\title{
A utilização do HBIM na documentação, na gestão e na preservação do Patrimônio Arquitetônico
}

\author{
The Use of HBIM on Documentation, Management and Conservation of the Architectural \\ Heritage
}

\author{
Mônica Martins Andrade Tolentino \\ UFBA, Brasil \\ Monica.andrade@ict.ufvjm.edu.br
}

\begin{abstract} model with a level of detail appropriate for the Church.

\section{Introdução}

O cadastro de um bem cultural tem como finalidade conservar a imagem e a história deste, visando a sua preservação. No Brasil, grande parte dos bens de valor histórico ainda não foi devidamente documentada. Mediante a ausência de um sistema de cadastro e gestão do patrimônio arquitetônico que utilize as tecnologias digitais de forma adequada, propõe-se a utilização do HBIM (Historic Building Information Modeling), afim de tornar o processo de documentação mais ágil, a gestão dos bens mais eficaz e a preservação do Patrimônio Arquitetônico mais efetiva.
\end{abstract}

This paper studies the HBIM concept and proposes a digital model of the Church of Bom Jesus de Matosinhos, in the city of Serro, aiming at its preservation. For this purpose, it (1) investigates and defines what information should be incorporated into the geometric model (attributes), so that it can be useful during the life cycle of the building and can contribute to the analysis and conservation of the object it represents; (2) defines the level of detail (LOD) to be used in HBIM models; (3) builds a HBIM

Keywords: HBIM; $\quad$ Architectural Heritage; $\quad$ Documentation; $\quad$ Management;

O Building Information Modeling (BIM) pode ser definido como uma metodologia avançada de trabalho colaborativo, que usa um modelo criado a partir de informações coordenadas e consistentes. Enquanto as ferramentas CAD focam apenas o projeto e a representação da edificação, o paradigma BIM pretende dar conta do ciclo de vida da edificação, e este é um aspecto que se presta muito bem ao patrimônio arquitetônico na medida em que permite cadastrálo, estudá-lo, acompanhá-lo e mantê-lo para as futura gerações. Além disto, o BIM possui outras excelentes características, tais como: visualização 3D e animações, a automação da produção de documentos digitais (perspectivas, projeções ortográficas, cortes, seções, detalhes) e relatórios de avaliação de desempenho estrutural, ambiental, energético e custos.

O Historic Building Information Modeling (HBIM) nada mais é que a extensão do conceito BIM para as edificações históricas e considerando as suas particularidades. Segundo Dore e Murphy (2012, p. 369), o HBIM contempla um processo que envolve uma solução de engenharia reversa onde, inicialmente, os elementos arquitetônicos são levantados usando um laser scanner ou fotogrametria. Em seguida, os dados levantados são combinados a objetos paramétricos, gerando o modelo completo da edificação. $O$ produto final HBIM é um modelo geométrico 3D completo, incluindo detalhes do objeto como materiais e métodos construtivos. A partir daí, cortes, detalhes, projeções ortográficas e perspectivas poderão ser gerados, baseando a análise e conservação de objetos históricos, estruturas e ambientes.

\section{Procedimentos Metodológicos}

Neste artigo, será estudado o conceito HBIM e proposto um modelo digital da Igreja do Bom Jesus de Matosinhos, na cidade do Serro, em Minas Gerais, visando a sua documentação. Para tanto, buscar-se-á:

- Investigar e definir quais informações deverão ser incorporadas ao modelo geométrico (atributos), para que o mesmo possa ser útil durante o ciclo de vida da edificação e possa contribuir para a análise e a conservação do objeto que representa;

- Definir o nível de detalhe (LOD, Level of Detail) a ser utilizado no modelo HBIM da Igreja do Bom Jesus de Matosinhos;

- Construir um modelo HBIM com nível de detalhe adequado para a Igreja do Bom Jesus de Matosinhos.

\section{Cadastro}

Oliveira (2008), alerta: "Um dos instrumentos importantes para a preservação da memória é o seu registro iconográfico, quer pelos métodos milenares, quer pelos processos e instrumentos mais recentes que a ciência e a técnica do nosso tempo nos trouxeram. Neste caso, desaparecido o objeto que testemunha o nosso passado, a sua imagem pode substituir, embora parcialmente, a necessidade imanente à natureza humana de manter contato com o que 
se foi. Daí uma das várias utilidades das representações cadastrais como forma de preservação da memória." (p. 13)

Além disso, o cadastro feito com apuro e exatidão é a base sobre a qual serão elaborados os projetos de intervenção, além de informar a evolução do bem, suas transformações e deformações ao longo do tempo. Oliveira (2008) ainda defende: "Para aqueles que se ocupam da análise históricocrítica do monumento, os cadastros são de primordial importância, pois podem permitir a leitura e o entendimento das corretas proporções do projeto original e descobrir eventuais traçados reguladores que comandaram a concepção da arquitetura, perfeitamente resgatáveis a partir de uma boa representação." (p. 13)

O autor ainda revela que o levantamento cadastral não é uma operação que se encerra com o levantamento rigoroso da geometria do bem, mas deve sofrer atualizações a cada momento em que é encontrada uma informação nova.

A partir da experiência como Secretária de Obras da Prefeitura Municipal de Serro e da Coordenação do Programa Monumenta na mesma cidade, além da pesquisa junto aos órgãos competentes (IPHAN e IEPHA), ficou evidenciado que não existe normatização com relação às técnicas e tecnologias utilizadas para o levantamento cadastral de bens de interesse histórico. Entretanto, alguns esforços vêm sendo feitos neste sentido.

Beirão (2011) cita a criação, pela Sociedade Internacional de Fotogrametria e Sensoriamento Remoto (ISPR) e pelo Conselho Internacional de Monumentos e Sítios (ICOMOS), do Comitê Internacional para Documentação de Patrimônio Cultural (CIPA), visando à excelência na medição, documentação e monitoramento dos bens, além do armazenamento adequado destas informações.

No Brasil, o IPHAN (Instituto do Patrimônio Histórico e Artístico Nacional) vem desenvolvendo e testando o Sistema Integrado de Conhecimento e Gestão (SICG), criado para integrar os dados sobre o Patrimônio Cultural do Brasil. O sistema está baseado em Word e Excell, mas o IPHAN vem trabalhando para a construção de um sistema informatizado, cujos usuários serão, também, os estados, municípios e entidades parceiras (como universidades, centros de estudo, museus, e outros).

Além disso, em 2013, o IPHAN, publicou o documento intitulado "Orientações para elaboração do projeto básico para contratação de projetos", documento esse que tem por objetivo orientar os procedimentos de elaboração do projeto básico para contratação de projetos de restauração, conservação, adequação para alteração de uso e outras intervenções no Patrimônio Edificado e de Espaços Públicos Urbanos (IPHAN, 2013).

$\mathrm{Na}$ Bahia, vem acontecendo uma ação com o apoio das instituições responsáveis pela preservação dos bens culturais no estado e da Faculdade de Arquitetura da Universidade Federal da Bahia (FAUFBA). O objetivo é a implantação de um Centro de Documentação do Patrimônio Arquitetônico e de Bens Integrados, ligado ao Laboratório de estudos avançados em Cidade, Arquitetura e tecnologias Digitais (LCAD), da Faculdade de Arquitetura da Universidade Federal da Bahia (FAUFBA). O LCAD visa ao desenvolvimento e apropriação do uso de tecnologias digitais que buscam aprimorar e agilizar o registro documental de bens arquitetônicos e integrados. No artigo intitulado "Um centro de documentação do patrimônio arquitetônico", os autores Amorim, Groetelaars e Lins (2008) defendem a criação do Centro e explicam: "Sob o aspecto das tecnologias utilizadas, o Centro estará estruturado em torno da pesquisa, da aplicação, da difusão de tecnologias digitais de ponta para o levantamento, o processamento, o armazenamento e a divulgação deste acervo arquitetônico. Dentre as principais tecnologias para o levantamento e o processamento dos dados provenientes dos acervos arquitetônicos, destacam-se a Medição Direta, a Modelagem Geométrica Tridimensional, a Fotogrametria Digital, o 3D Laser Scanning, os Sistemas de Informações Geográficas e os Sistemas Hipermídia." (p.201)

Tomando como exemplo o que vem acontecendo na Bahia, este artigo visa estudar os métodos citados, de modo particular o HBIM. A criação de um modelo HBIM para a Igreja Bom Jesus de Matosinhos buscará demonstrar, experimentalmente, qual nível de detalhe deverá ser utilizado nos modelos HBIM e quais informações deverão ser incorporadas a este modelo, para que o mesmo possa ser útil durante todo o ciclo de vida da edificação e possa basear a análise e a conservação do edifício.

\section{Atributos}

Para fins de cadastro, o Instituto do Patrimônio Histórico e Artístico Nacional (IPHAN, 2013), órgão do Ministério da Cultura que tem a missão de preservar o patrimônio cultural brasileiro, exige a "[...] descrição física do bem objeto de intervenção, características de uso, estado geral de preservação e conservação. Descrição histórica de construção e ocupação do bem. Referência a relatórios técnicos do IPHAN ou outras entidades."

O IPHAN (2013) entende que a elaboração de um projeto de restauração deve ser precedida de um estudo sobre o monumento sob diversos pontos de vista, visando compreender o seu significado atual e ao longo do tempo, sua evolução e os valores pelos quais foi reconhecido como patrimônio cultural. Este levantamento, que o IPHAN denomina Identificação e Conhecimento do Bem, engloba:

- Pesquisa histórica: que considera aspectos políticos, socioeconômicos, técnicos e artísticos, objetivando conhecer o bem e situá-lo no tempo;

- Plano de levantamentos e Prospecções: contendo o cronograma dos serviços, mapeamentos de instalações e equipamentos; 
- Levantamento físico: que abrange (a) o levantamento de dados cartoriais e jurídicos, (b) o levantamento cadastral, (c) - levantamento topográfico, (d) o levantamento e mapeamento físico e paisagístico;

- Análise tipológica, identificação de materiais e sistema construtivo: contendo (a) prospecções arquitetônica, estrutural e do sistema construtivo, arqueológica e de bens integrados, (b) diagnóstico, com análise do estado de conservação nos aspectos construtivos, estéticos e de mapeamento de danos.

Posto isso, percebe-se que a geração de um modelo puramente geométrico comporta somente uma pequena parte daquilo que é esperado para fins de cadastro do patrimônio histórico. A utilização de ferramentas HBIM possibilita, além da criação do modelo geométrico, a inserção de atributos mais específicos, como os já citados materiais, métodos construtivos, estado de conservação, mapeamento de danos e evolução ao longo do tempo.

\section{Definições de LOD}

\section{Nível de Desenvolvimento (LOD, Level of Development)}

O nível de desenvolvimento (LOD) é uma referência que possibilita aos profissionais da indústria de Arquitetura e Construção especificar e articular com um alto nível de clareza e confiabilidade o conteúdo dos Modelos de Informação da Construção (BIMs) em vários estágios do processo de concepção e construção. Esta articulação clara permite aos autores do modelo definirem em que situação seus modelos podem ser usados, e permite que os usuários entendam claramente a função e as limitações dos modelos que estão recebendo.

Baseado nos conceitos criados em 2008 e atualizados em 2013 pelo American Institute of Architects (AIA), o BIMForum (2013) publicou o documento Level of Development Specification For Building Information Models, onde define:

- No LOD 100 os elementos do modelo podem ser representados por um símbolo ou uma representação genérica e não são representações geométricas. Qualquer informação derivada destes modelos deve ser considerada aproximada.

- No LOD 200 os elementos do modelo podem ser representados graficamente como um objeto ou como um sistema genérico, com quantidades, tamanho, forma, localização e orientação aproximadas. Informações não gráficas podem ser anexadas a ele. Neste nível, os elementos podem ser reconhecidos como os componentes que eles representam. Qualquer informação derivada destes modelos deve ser considerada aproximada.

- No LOD 300 os elementos são representados graficamente de forma precisa e informações não gráficas podem ser anexadas a ele. Sua quantidade, tamanho, forma localização e orientação podem ser medidos diretamente no modelo.

- No LOD 350 os elementos são representados graficamente de forma precisa, com relação à quantidade, tamanho, forma, localização, orientação e interface com outros sistemas, informações estas que podem ser aferidas diretamente no modelo. Neste nível, são modelados os itens necessários à coordenação entre disciplinas, à compatibilização de projetos e à verificação de interferências. Informações não gráficas podem ser anexadas a ele.

- No LOD 400 os elementos são representados graficamente de forma detalhada e precisa, visando a fabricação. Informações não gráficas podem ser anexadas. Quantidade, tamanho, forma, localização, orientação e informações relacionadas à fabricação, instalação e execução, podem ser aferidas diretamente no modelo.

\section{Nível de Detalhe (LOD, Level of Detail)}

Em computação gráfica, o nível de detalhe (LOD) é uma técnica que permite várias representações para um mesmo objeto, que vão sendo ativadas de acordo com as diferentes distâncias a que o utilizador se encontra. Ou seja, envolve o decréscimo da complexidade da representação de objetos 3D, permitindo economizar recursos de computação. A utilização das técnicas de LOD permite um aumento da eficiência e rendimento, diminuindo assim o nível de trabalho.

Tendo como objetivo a criação do modelo BIM da Capela de Nossa senhora da Escada a partir de nuvem de pontos, visando o estabelecimento de métodos e procedimentos de modelagem adequados a diferentes níveis de detalhes e aplicações, permitindo associar as características específicas de determinados elementos construtivos à escala da representação gráfica, técnica de levantamento adotada e finalidade do modelo e após estudar e avaliar a terminologia em diversos livros e guias sobre documentação arquitetônica (English Heritage, 2006; Letellier, 2007; Cramer; Breitling, 2007; East, 2013; GSA, 2009; Plowman, 2012), Groetelaars (2015) elaborou um quadro a ser usado como referência para a modelagem BIM. Nele são propostos cinco níveis de detalhe, que associam a precisão do modelo à sua finalidade.

- No Nível 1 deve ser representada a volumetria externa, contemplando paredes e coberturas, objetivando 0 planejamento inicial do projeto de documentação, a identificação de áreas prioritárias, a visualização da edificação no contexto urbano e a utilização em sistemas de informações geográficas.

- No Nível 2 os principais elementos construtivos do exterior e interior devem ser modelados de forma simplificada: paredes, pisos, escadas, pilares, forros, e aberturas de portas e janelas. Devem ser executados, ainda: a modelagem do terreno, a identificação das paredes (interior e exterior) e a inclusão dos parâmetros sobre desvios de modelagem. Tal modelo será útil para a estimativa de áreas 
e volumes com diversas finalidades, como manutenção e estudo de viabilidade

- No Nível 3 deverão ser incluídos os detalhes de elementos básicos, usando preferencialmente as famílias existentes. Famílias em baixo nível de detalhe poderão ser criadas, quando necessário. Este modelo poderá ser exportado para programas de gerenciamento de facilities, poderá ser usado no planejamento funcional e como base para pequenas intervenções na edificação.

- No Nível 4 deverão ser incluídos elementos detalhados e mobiliário. Famílias com médio nível de detalhe deverão ser criadas. Materiais de acabamento dos principais elementos construtivos deverão ser descritos. Uma nova modelagem dos elementos com desvios superiores aos máximos deverá ser feita. $O$ modelo assim criado servirá para documentação arquitetônica de edificações de interesse histórico-cultural e como base para projetos de intervenção.

- No Nível 5 deverão ser criadas famílias em alto nível de detalhe, as informações semânticas associadas aos elementos construtivos deverão ser inseridas, os arquivos de imagens deverão ser associados e texturas foto realísticas em trechos relevantes deverão ser aplicadas. O modelo servirá para fins de documentação arquitetônica de edificações de interesse histórico-cultural, monitoramento estrutural e como base para projetos de intervenção mais complexos.

\section{Modelo}

Tendo em vista o que foi discutido nas seções anteriores, cabem ainda algumas considerações.

Não se deve confundir o nível de desenvolvimento (LOD, Level of Development) com nível de detalhe (LOD, Level of Detail). Enquanto o primeiro está relacionado ao grau de maturidade e confiabilidade no desenvolvimento de modelos $\mathrm{BIM}$, o segundo se refere à quantidade de detalhes incluídas nos elementos do modelo. Neste artigo, objetiva-se a criação de um modelo BIM para o cadastro de um monumento histórico existente (modelo HBIM), e não de um projeto de intervenção neste monumento. Sendo assim, LOD, aqui, será entendido como nível de detalhe.

O nível de detalhe pretendido para o modelo HBIM a ser modelado para a Igreja de Bom Jesus do Matozinhos, Nível 5 segundo a classificação proposta por Groetelaars (2015), foi determinado levando-se em consideração os produtos exigidos pelo IPHAN (2013).
Salienta-se que o modelo final contemplará apenas os bens imóveis, excluindo-se os elementos artísticos. Isto porque este tipo de bem, como estátuas e objetos decorativos, exigem outras técnicas de modelagem não suportadas pelas ferramentas BIM tradicionais.

\section{A Igreja de Bom Jesus do Matozinhos}

Implantada numa parte baixa da cidade do Serro (ao contrário das outras igrejas locais), a Igreja do Bom Jesus do Matozinhos demonstra um feitio cuidadoso, onde se cuidou principalmente dos detalhes da ornamentação. As pinturas murais e principalmente o forro da capela-mor qualificam a singularidade dos trabalhos de um importante pintor atuante na região: Silvestre de Almeida Lopes. Reconhecidamente um monumento arquitetônico de sumo valor para a comunidade local, a igreja encontra-se tombada pelo IPHAN desde 1944. (IPHAN, 2005)

A construção da Igreja do Bom Jesus de Matozinhos é estimada entre o final da década de 1770 e início da de 1780. Seu adro é acessado por escadaria de pedra

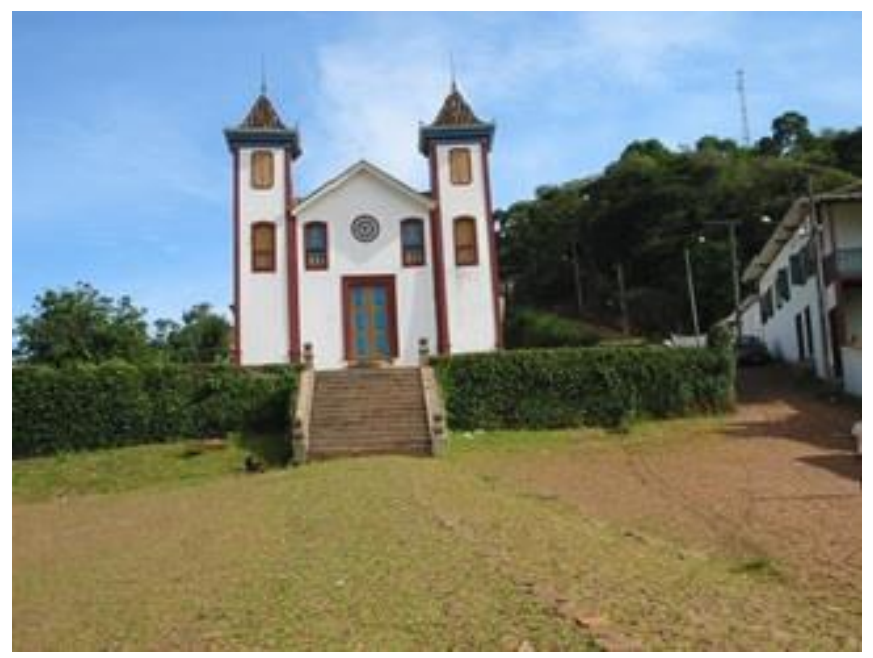

trabalhada e circundado por muro de arrimo, também de pedra. O piso mantém-se original, de calçamento pé-demoleque. A fachada apresenta torres de seção quadrada com telhados arqueados e frontão simples, de linhas retas com óculo redondo envidraçado. Há uma escadaria de pedra com degraus em arco, ligando o adro ao piso do átrio.

Figura 1: Fachada Frontal da Igreja Bom Jesus do Matozinhos. Fonte: Teixeira (2003)

A planta tem partido simples, dividindo-se em nave, capelamor e, ao longo das suas paredes, os anexos laterais correspondentes às sacristias em corredor. Construída em taipa e madeira, possui cobertura em duas águas em telha cerâmica. 


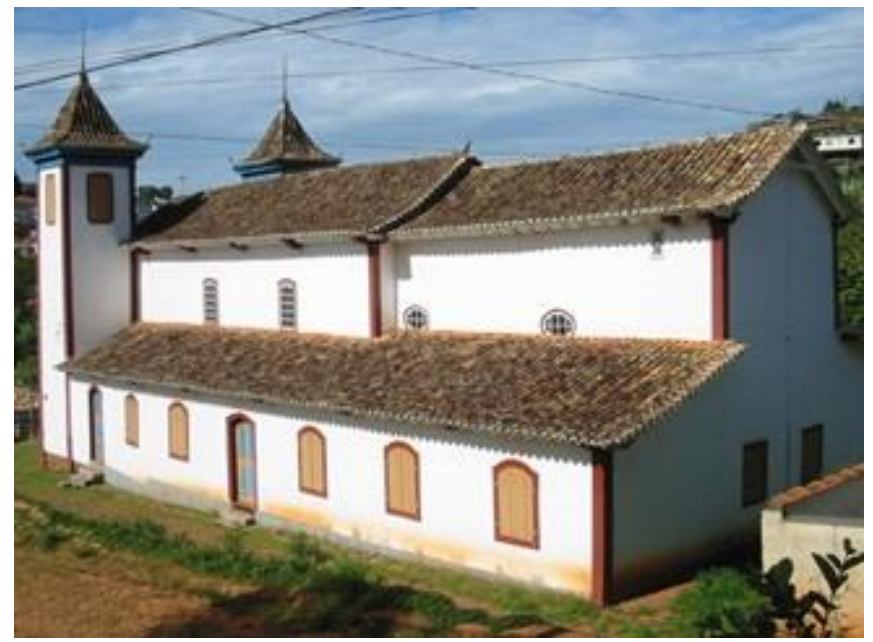

Figura 2: Fachada lateral direita da Igreja Bom Jesus do Matozinhos. Fonte: Teixeira (2003)

Internamente, apresenta pisos de tabuado largo, forros abobadados em tabuado liso, cimalhas de madeira e coro simples com balaustrada de madeira. (FUNDAÇÃO JOÃO PINHEIRO, 1995)

Tendo sido restaurada entre 2007 e 2008, a igreja encontrase atualmente em bom estado de conservação. A intervenção teve como principal objetivo recuperar a integridade estrutural da construção, substituindo peças corroídas e fortalecendo fundação, piso, forro e telhado. Um minucioso trabalho garantiu ainda a revitalização dos elementos artísticos, recuperando imagens e painéis característicos do rococó. A igreja também recebeu segurança extra, como a instalação de para-raios e sistemas de alarme e de drenagem. As obras foram feitas através do programa Monumenta, fruto de uma parceria entre Governo Federal (por meio do Ministério da Cultura) e Banco Interamericano de Desenvolvimento (BID), com apoio da Organização das Nações Unidas para a Educação, Ciência e Cultura (Unesco). (IEPHA, 2008)

\section{Levantamento Cadastral por Medição Direta}

Como pré-requisito para a elaboração do projeto para a restauração da Igreja em 2007, foi feito um levantamento cadastral com uso de trena e máquina fotográfica. Este resultou em cinco pranchas no formato $\mathrm{A} 1$, que contemplam: (1) plantas de implantação e cobertura, (2) planta térreo e corte AA, (3) planta do coro e corte BB, (4) fachadas sudoeste/nordeste e (5) fachadas laterais. Outras cinco pranchas, correspondentes às primeiras, mostram 0 mapeamento de danos/estado de conservação à época e foram a base para a criação do modelo HBIM em Revit.

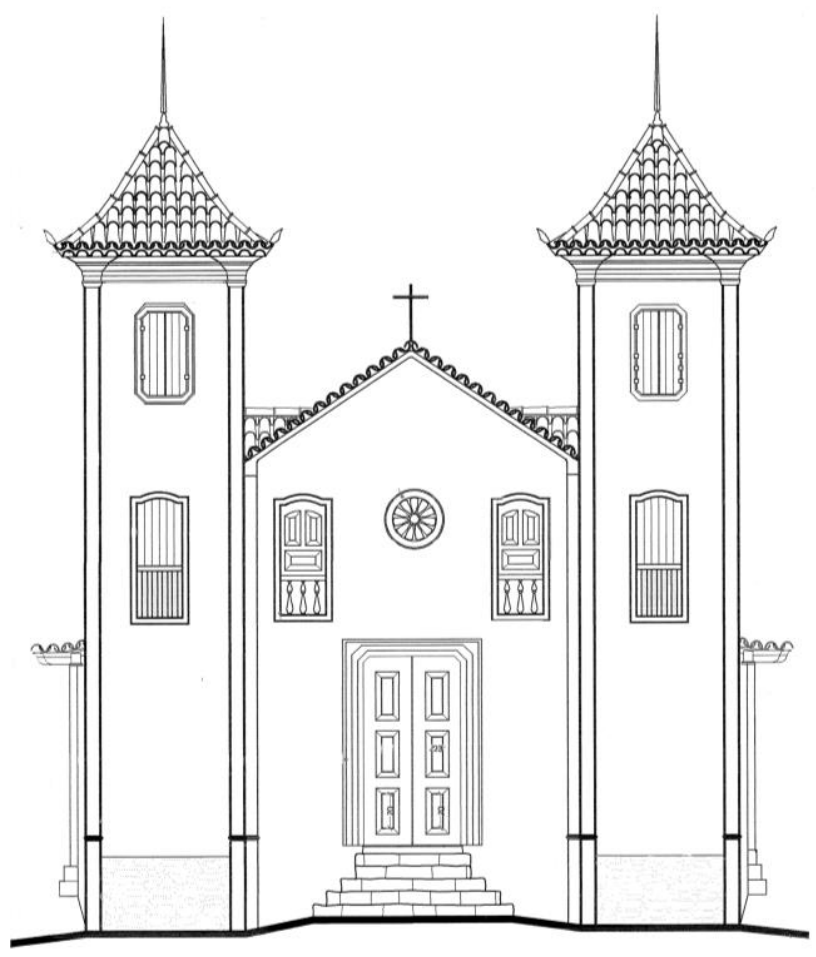

Figura3: Fachada Sudoeste da Igreja Bom Jesus do Matozinhos. Fonte: Teixeira (2006)

\section{Levantamento Cadastral por Varredura à Laser}

Foi realizado, no dia 12/06/2015, o levantamento da igreja por varredura à laser. Para tanto, foi utilizado um scanner Faro Focus 3D e seus respectivos acessórios (esferas, cartão de memória e fonte de alimentação. Foram escaneadas 46 cenas em aproximadamente 10 horas de trabalho de campo. Posteriormente, utilizou-se os softwares Faro Scene e Autodesk Recap para o registro e a limpeza da nuvem de pontos. 


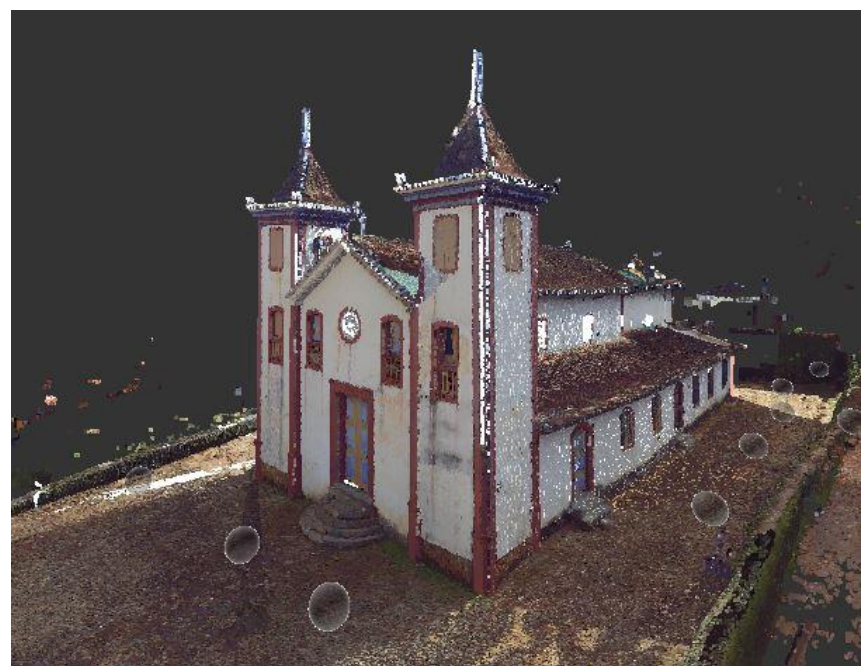

Figura 4: Nuvem de pontos da Igreja Bom Jesus do Matozinhos. Fonte: Tolentino (2016)

Este levantamento será usado, oportunamente, para verificar a precisão do modelo HBIM elaborado a partir do levantamento feito por medição direta em 2006 e basear as correções necessárias.

\section{Modelo Geométrico}

O modelo geométrico da edificação está sendo desenvolvido com a utilização do software Revit 2016, da Autodesk. Paredes, pisos e telhados estão sendo gerados com as ferramentas padrão, enquanto famílias de janelas e portas estão sendo especialmente criadas no Editor de Famílias para este modelo.

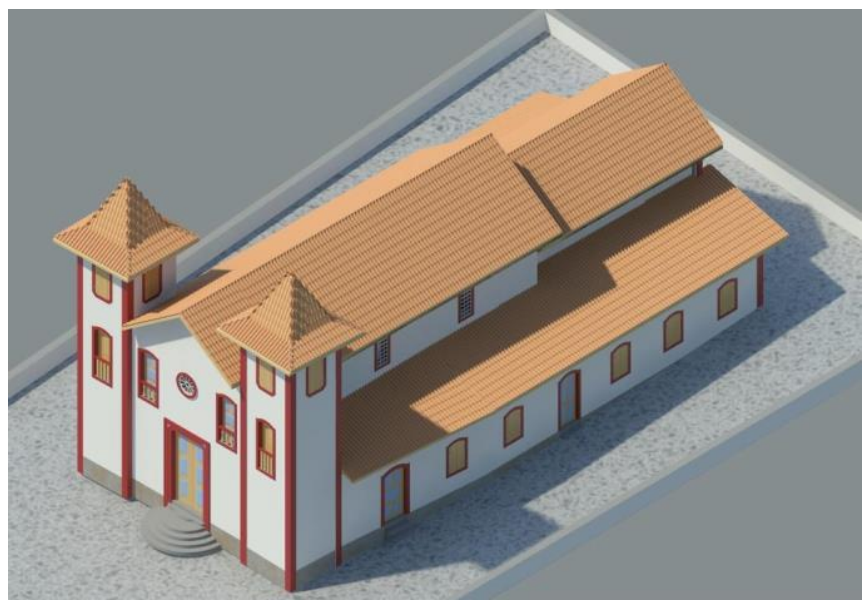

Figura 5: Modelo da Igreja Bom Jesus do Matozinhos desenvolvido no Revit. Fonte: Tolentino (2016)
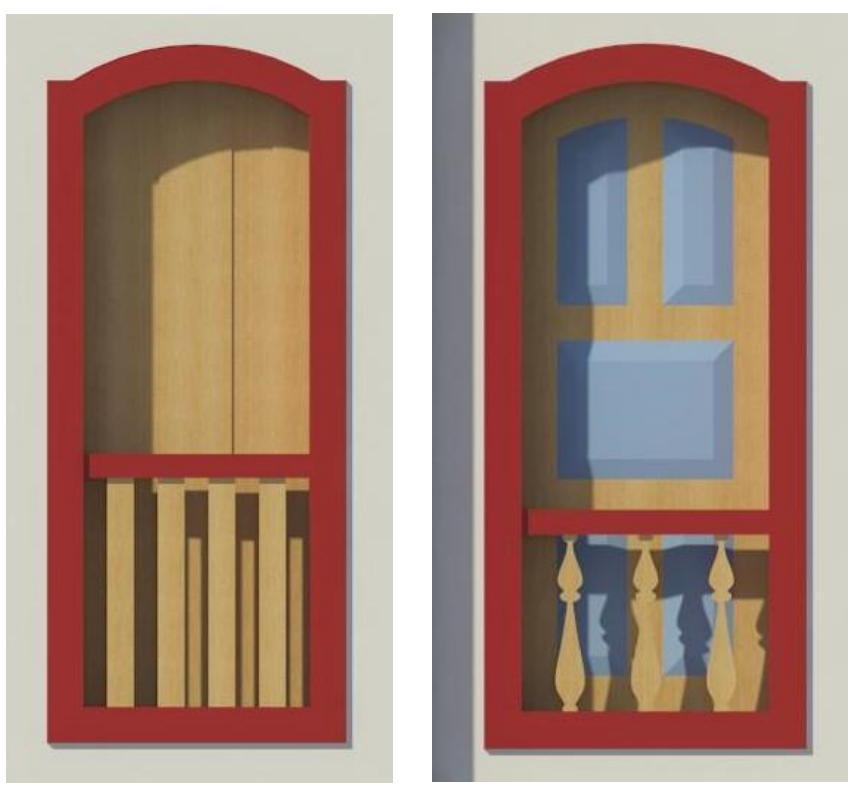

Figura 6: Famílias de portas-balcão criadas no editor de famílias do Revit. Fonte: Tolentino (2016)

\section{Atributos}

É possível adicionar metadados às propriedades Tipo ou Instância de uma família no Editor de família e no ambiente do projeto.

Os parâmetros padrão são fornecidos para os atributos de metadados típicos de uma família, como o fabricante, modelo, descrição e custo, mas é possível adicionar parâmetros conforme necessário. Estes dados podem ser incluídos em tabelas.

Quadro 1: Tipos de Parâmetros Padrão de Famílias do Revit. Fonte: Autodesk, 2016

\begin{tabular}{|c|c|}
\hline Nome & Descrição \\
\hline Texto & $\begin{array}{l}\text { Completamente personalizável. Pode ser usado } \\
\text { para coletar dados únicos. }\end{array}$ \\
\hline Inteiro & $\begin{array}{l}\text { Um valor é sempre expresso como um número } \\
\text { inteiro. }\end{array}$ \\
\hline Número & $\begin{array}{l}\text { Usado para coletar dados numéricos diversos. } \\
\text { Pode ser definido por uma fórmula. Também } \\
\text { pode haver números reais. }\end{array}$ \\
\hline Comprimento & $\begin{array}{l}\text { Pode ser utilizado para estabelecer } \\
\text { comprimento de um elemento ou } \\
\text { subcomponente. Pode ser definido por uma } \\
\text { fórmula. Este é o tipo padrão. }\end{array}$ \\
\hline Área & $\begin{array}{l}\text { Pode ser utilizada para estabelecer a área de um } \\
\text { elemento ou subcomponente. As fórmulas } \\
\text { podem ser usadas neste campo. }\end{array}$ \\
\hline Volume & $\begin{array}{lllll}\text { Pode ser utilizado para estabelecer } & \text { o } \\
\text { comprimento de um elemento } & \text { ou } \\
\text { subcomponente. As fórmulas podem } & \text { ser } \\
\text { utilizadas neste campo. } & & \\
\end{array}$ \\
\hline Ângulo & $\begin{array}{l}\text { Pode ser utilizado para estabelecer o ângulo de } \\
\text { um elemento ou subcomponente. As fórmulas } \\
\text { podem ser utilizadas neste campo. }\end{array}$ \\
\hline Inclinação & $\begin{array}{l}\text { Pode ser usado para criar parâmetros que } \\
\text { definem a inclinação. }\end{array}$ \\
\hline Moeda & Podem ser usadas para criar parâmetros de \\
\hline
\end{tabular}




\begin{tabular}{|c|c|}
\hline & moeda. \\
\hline $\begin{array}{l}\text { Densidade } \\
\text { de Massa }\end{array}$ & $\begin{array}{l}\text { Um valor que representa a massa por unidade } \\
\text { de volume de um material. }\end{array}$ \\
\hline URL & $\begin{array}{l}\text { Fornece um link da Web para o URL definido } \\
\text { pelo usuário. }\end{array}$ \\
\hline Material & $\begin{array}{l}\text { Estabelece o parâmetro ao qual um material } \\
\text { específico pode ser atribuído. }\end{array}$ \\
\hline Imagem & $\begin{array}{l}\text { Estabelece um parâmetro no qual uma } \\
\text { determinada imagem raster pode ser atribuída. }\end{array}$ \\
\hline Sim/Não & $\begin{array}{l}\text { Usado com maior freqüência para propriedades } \\
\text { de instância quando o parâmetro é definido como } \\
\text { um Sim ou Não. }\end{array}$ \\
\hline $\begin{array}{l}\text { Texto de } \\
\text { múltiplas } \\
\text { linhas }\end{array}$ & $\begin{array}{l}\text { Estabelece um parâmetro no qual as sequências } \\
\text { de texto de múltiplas linhas mais longas podem } \\
\text { ser utilizadas. Clique no botão Procurar na paleta } \\
\text { Propriedades para inserir sua sequência de } \\
\text { caracteres de texto. }\end{array}$ \\
\hline $\begin{array}{l}\text { Tipo de } \\
\text { superfície } \\
\text { dividida }\end{array}$ & $\begin{array}{l}\text { Estabelece um parâmetro com o qual os } \\
\text { componentes da superfície dividida (como } \\
\text { painéis e padrões) podem ser controlados. As } \\
\text { fórmulas podem ser utilizadas neste campo. Este } \\
\text { parâmetro está disponível somente para as } \\
\text { famílias de massa. }\end{array}$ \\
\hline
\end{tabular}

Para as portas-balcão mostradas na figura 6 , por exemplo, foram criados os seguintes parâmetros:

Tabela 2: Tipos de parâmetros adicionados para a família janela. Fonte: Tolentino, 2016

\begin{tabular}{|ll|}
\hline Nome & Descrição \\
\hline $\begin{array}{l}\text { Estado de } \\
\text { Preservação }\end{array}$ & $\begin{array}{l}\text { O parâmetro pode ser escolhido como } \\
\text { Integro, Pouco Alterado, Muito Alterado ou } \\
\text { Descaracterizado }\end{array}$ \\
\hline $\begin{array}{l}\text { Estado de } \\
\text { Conservação }\end{array}$ & $\begin{array}{l}\text { O parâmetro pode ser escolhido como Bom, } \\
\text { Precário, Em Arruinamento ou Arruinado }\end{array}$ \\
\hline $\begin{array}{l}\text { Ataque de } \\
\text { insetos xilófagos }\end{array}$ & Sim/Não \\
\hline $\begin{array}{l}\text { Ataque de Sim/Não } \\
\text { fungos }\end{array}$ & Sinarnato \\
\hline
\end{tabular}

\section{Resultados e Discussão}

No Brasil, a proposta de aplicação da ferramenta HBIM tem os objetivos, além da modelagem geométrica, de incorporar o armazenamento de atributos (como materiais e técnicas construtivas utilizados, estado de conservação, etc.) e de dados de diferentes naturezas (como arquivos de texto, documentos capturados por scanner, fotografias digitais, entre outros) e fornecer instrumentos para a gestão do patrimônio histórico. Estes dados poderão, oportunamente, basear projetos de intervenção, bem como a operação e a manutenção dos edifícios, o que contribuirá para a preservação mais eficaz do Patrimônio Histórico no Brasil.

\section{Agradecimentos}

À arquiteta Letícia Miguel Teixeira por disponibilizar o levantamento cadastral da Igreja Bom Jesus do Matozinhos, realizado no ano de 2006.

A Carlos Souza, gerente de engenharia da empresa Minerium, localizada em Belo Horizonte, por realizar e ceder o levantamento com o uso de laser scanner da Igreja Bom Jesus de Matosinhos.

\section{Referências}

AMORIM, Arivaldo Leão de; GROETELAARS, Natalie J.; LINS, Eugênio de A. (2008). Um centro de documentação do patrimônio arquitetônico. Fórum do Patrimônio, v.2, n.1. Disponível

http://www.forumpatrimonio.com.br/seer/index.php/forum_patrim onio/article/view/98

AUTODESK (2016). Parâmetros comuns de família. Disponível em: http://help.autodesk.com/view/RVT/2016/PTB/?guid=GUIDACDC1C99-0278-4FC8-9E42-19659D7534F9.

BAIAK, A., YAAGOUBI, R., BOEHM, J. (2015). Integration of Jeddah Historical BIM and 3D GIS for documentation and restoration of historical monument. The International Archives of the Photogrammetry, Remote Sensing and Spatial Information Sciences, XL-5/W7, 29-34. doi: 10.5194. Disponível em http://www.int-arch-photogramm-remote-sens-spatial-inf-sci.net/ $\mathrm{XL}-5-\mathrm{W} 7 /$ index.html

BARAZZETTI, L., BANFI, F., BRUMANA, R, ORENI, D., PREVITALI, M., RONCORONI, F. (2015) HBIM and augmented information: Towards a wider user community of image and range-based reconstructions. The International Archives of the Photogrammetry, Remote Sensing and Spatial Information Sciences, XL-5/W7, 35-42. doi: 10.5194. Disponível em http://www.int-arch-photogramm-remote-sens-spatial-inf-sci.net/ XL-5-W7/index.html

BEIRÃO, C. (2011). O Potencial do Laser Scanner Terrestre para o Inventário do Patrimônio Arquitetônico. Dissertação (Mestrado em Engenharia Civil) - Florianópolis: Universidade Federal de Santa Catarina,

BOITO, C. (2002). Os restauradores ( $2^{\mathrm{a}}$ edição). São Paulo: Ateliê Editorial.

BRANDI, C. (2013). Teoria da Restauração (4⿳亠丷厂 edição). São Paulo: Ateliê Editorial.

CARBONARA, G. (2013). Apresentação. In: BRANDI, Cesare. Teoria da Restauração (4⿳亠丷a edição). São Paulo: Ateliê Editorial.

CHENG, H-M., YANG, W-B., YEN, Y-N. (2015). BIM applied in historical building documentation and refurbishing. The International Archives of the Photogrammetry, Remote Sensing and Spatial Information Sciences, XL-5/W7, 85-90. doi: 10.5194. Disponível em http://www.int-arch-photogramm-remote-sensspatial-inf-sci.net/ XL-5-W7/index.html

CHOAY, F. (2006). A alegoria do patrimônio (3a edição). São Paulo: Estação Liberdade - Ed. UNESP.

CRAMER, J., BREITLING, S. (2007). Architecture in existing fabric: Planning, Design, Building. Berlim: Birkhauser.

DE MASI, A. (2015). Survey guidelines and its reading criteria for monitoring and transmitting cultural heritage values. The International Archives of the Photogrammetry, Remote Sensing and Spatial Information Sciences, XL-5/W7, 113-121. doi: 10.5194. Disponível em http://www.int-arch-photogramm-remotesens-spatial-inf-sci.net/ XL-5-W7/index.html

DOURADO, O. (2010) Arquiteturas Modernas: A fugacidade como espírito. 19\&20, v.V, n.3. Disponível em: http://www. dezenoveevinte.net/arte\%20decorativa/ad_odete.htm.

DOURADO, O. (2003). Por um restauro urbano: novas edificações que restauram cidades monumentais. RUA - Revista de Urbanismo e Arquitetura, v.1, n.8, 8-13. 
DOURADO, O. (1989). Para sempre, memória. RUA - Revista de Arquitetura e Urbanismo, v.2, n.3, 65-74.

DORE, C., MURPHY, M. (2012). Integration of Historic Building Information Modeling and 3D GIS for Recording and Managing Cultural Heritage Sites. 18th International Conference on Virtual Systems and Multimedia: "Virtual Systems in the Information Society", 369-376.

EAST, B., CARRASQUILLO-MANGUAL, M. (2013). The COBie Guide: a commentary to the NBIMS-US COBie standard. Disponível em file://D:/Downloads/COBie_Guide-Public_ Release_3.pdf

ENGLISH HERITAGE. (2006) Understanding Historic Buildings: a guide to good recording practice. Swindon: English Heritage.

FUNDAÇÃO JOÃO PINHEIRO (1995). Minas Gerais Monumentos Históricos e Artísticos - Circuito do Diamante (2 $2^{\mathrm{a}}$ edição). Belo Horizonte

GODOY, V., MOURA, A., MENEZES, P. (2010). A cartografia digital e navegação virtual na promoção do usuário como agente central na produção da representação do espaço. Revista Brasileira de Cartografia, n. 62/04. (ISSN 0560-4613)

GROETELAARS, N. (2015) Criação de modelos BIM a partir de nuvens de pontos: estudo de métodos e técnicas para documentação arquitetônica. Tese (Doutorado em Arquitetura). Salvador: Universidade Federal da Bahia, Faculdade de Arquitetura.

GRUSSENMEYER, P., BURENS, A., GUILLEMIN, S., ALBY, E., SIMONETTI, F., MARCHETTI, M. (2015). 3D recording methodology applied to the Grotta Scritta prehistoric rock-shelter in Olmeta-di-Capocorso (Corsica, France). The International Archives of the Photogrammetry, Remote Sensing and Spatial Information Sciences, XL-5/W7, 179-185. doi:10.5194. Disponível em http://www.int-arch-photogramm-remote-sensspatial-inf-sci.net/ XL-5-W7/index.html

GSA - General Services Administration. (2009) GSA Building Information Modeling Guide Series: 03 - GSA BIM Guide for 3D Imaging. Disponível em http://www.gsa.gov/portal/mediald/ 226819/fileName/GSA_BIM_Guide_Series_03.action.

HE, J., LIU, J., XU, S., Wu, C., ZHANG, J. (2015). A GIS-based cultural heritage study framework on continuous scales: a case study on 19th century military industrial heritage. The International Archives of the Photogrammetry, Remote Sensing and Spatial Information Sciences, XL-5/W7, 215,222. doi:10.5194. Disponível em http://www.int-arch-photogrammremote-sens-spatial-inf-sci.net/ XL-5-W7/index.html

HUYSEN, A. (2000). Seduzidos pela memória: arquitetura, monumentos, mídia. Rio de Janeiro: Aeroplano/MAM.

IEPHA. Igreja Bom Jesus de Matozinhos no Serro. Disponível em: http://www.iepha.mg.gov.br/banco-de-noticias/576-igreja-dosenhor-bom-jesus-de-matozinhos-no-serro.

IPHAN. Instituto do Patrimônio Histórico e Artístico Nacional. Orientações para elaboração do projeto básico para contratação de projetos. Disponível em: http://portal.iphan.gov.br/

uploads/ckfinder/arquivos/Orienta\%C3\%A7\%C3\%B5es\%20para \%20elabora\%C3\%A7\%C3\%A30\%20de\%20projeto\%20b\%C3\%A 1 sico.pdf.

IPHAN. Instituto do Patrimônio Histórico e Artístico Nacional. Sistema Integrado de Conhecimento e Gestão SICG. Disponível http://portal.iphan.gov.br/portal/montarPaginaSecao.do?id=1489 7\&retorno=paginalphan.
KUHL, B. (2006). História e Ética na Conservação e na Restauração de Monumentos Históricos. São Paulo: Revista CPC, v.1, n.1, 16-40.

KUHL, B. (2002). Os restauradores e o pensamento de Camillo Boito sobre a restauração. In: BOITO, C. Os restauradores ( $2^{\mathrm{a}}$ edição). São Paulo: Ateliê Editorial.

LANDES, T., KUHNLE, G., BRUNA, R. (2015). 3D modeling of the Strasbourg's cathedral basements for interdisciplinary research and virtual visits. The International Archives of the Photogrammetry, Remote Sensing and Spatial Information Sciences, XL-5/W7, 263-270. doi: 10.5194. Disponível em http://www.int-arch-photogramm-remote-sens-spatial-inf-sci.net/ XL-5-W7/index.html

LETELLIER, R. (2007). Recording, Documentation, and Information Management for the Conservation of Heritage Places: Guiding Principles. Los Angeles: J. Paul Getty Trust.

LI, K., LI, S., LIU, Y., WANG, W., WU, C. (2015) Coordination between understanding historic buildings and BIM modelling: a 3D-output oriented and typological data capture method. The International Archives of the Photogrammetry, Remote Sensing and Spatial Information Sciences, XL-5/W7, 283-288. Doi: 10.5194. Disponível em http://www.int-arch-photogramm-remotesens-spatial-inf-sci.net/ XL-5-W7/index.htm

MA, Y.-P., HSU, C.-C., LIN, M.-C., TSAI, Z.-W., CHEN, J.-Y. (2015). Parametric Workflow (BIM) for the repair construction of traditional historic architecture in Taiwan. The International Archives of the Photogrammetry, Remote Sensing and Spatial Information Sciences, XL-5/W7, 315-322. doi: 10.5194. Disponível em http://www.int-arch-photogramm-remote-sensspatial-inf-sci.net/ XL-5-W7/index.html

MONUMENTA (2005). Conjunto Arquitetônico e urbanístico da cidade do Serro. Perfil do Projeto. Caderno 04. Brasília.

MOURA, A. (2008). Geoprocessamento na Gestão do Patrimônio Histórico. Fórum Patrimônio: ambiente construído e patrimônio sustentável, v.2, n.2. Disponível em: http://www.forumpatrimonio. com.br/seer/index.php/forurpatrimonio/article/view/96

MURPHY, M.; MCGOVERNA, E.; PAVIA, S. (2011). Historical Building Information Modelling-Adding Intelligence to Laser and Image based surveys. The International Archives of the Photogrammetry, Remote Sensing and Spatial Information Sciences, XXXVIII-5/W16, 7. Disponivel em: http://www.int-archphotogramm-remote-sens-spatial-inf-sci.net/XXXVIII-5W16/1/2011/isprsarchives-XXXVIII-5-W16-1-2011.pdf

OLIVEIRA, M. (2008). A Documentação como Ferramenta de Preservação da Memória. Brasília, D.F: IPHAN / Programa Monumenta.

PLOWMAN CRAVEN LIMITED (2012). BIM Survey Specification and Reference Guide. Disponível em http://www.plowmancraven. co.uk/bim-survey-specification/

RAMOS, M., REMONDINO, F. (2015). Data fusion in cultural heritage - a review. The International Archives of the Photogrammetry, Remote Sensing and Spatial Information Sciences, XL-5/W7, 359-363. doi:10.5194. Disponível em http://www.int-arch-photogramm-remote-sens-spatial-inf-sci.net/ XL-5-W7/index.html

RIEGEL, A. (2006). O culto moderno dos monumentos: sua essência e sua gênese. Goiânia: Ed. Da UGG.

RUSKIN, John. (1996). A Lâmpada da Memória. Apresentação, Tradução e Comentários Críticos Odete Dourado. Salvador: UFBA, n. 2

SUN, Z., CAO, Y. (2015). Data processing worflowsfrow low-cost digital survey to various applications: three case studies of 
Chinese historic architecture. The International Archives of the Photogrammetry, Remote Sensing and Spatial Information Sciences, XL-5/W7, 409-416. doi:10.5194. Disponível em http://www.int-arch-photogramm-remote-sens-spatial-inf-sci.net/ $\mathrm{XL}-5$-W7/index.html

VIOLLET-LE-DUC, E. (2006). Restauração. São Paulo: Atelier Editorial.

VIOLLET-LE-DUC, E. (1996). Restauração. Apresentação, Tradução e Comentários Críticos Odete Dourado (3ª edição). Salvador: UFBA, n. 1.

VOLK, R., STENGEL, J., SCHULTMANN, F. (2014). Building Information Modeling (BIM) for existing Buildings - Literature
Review and Future Needs. Automation in Construction, V. 38, 109-127.

WIECZOREK, D. (2006). Introduction du traducteur. In: RIEGL, A. O culto moderno dos monumentos: sua essência e sua gênese. Goiânia: Ed. Da UGG. Please delete this line after your work is complete. It is just a reference to maintain the graphic balance of the
two columns. 